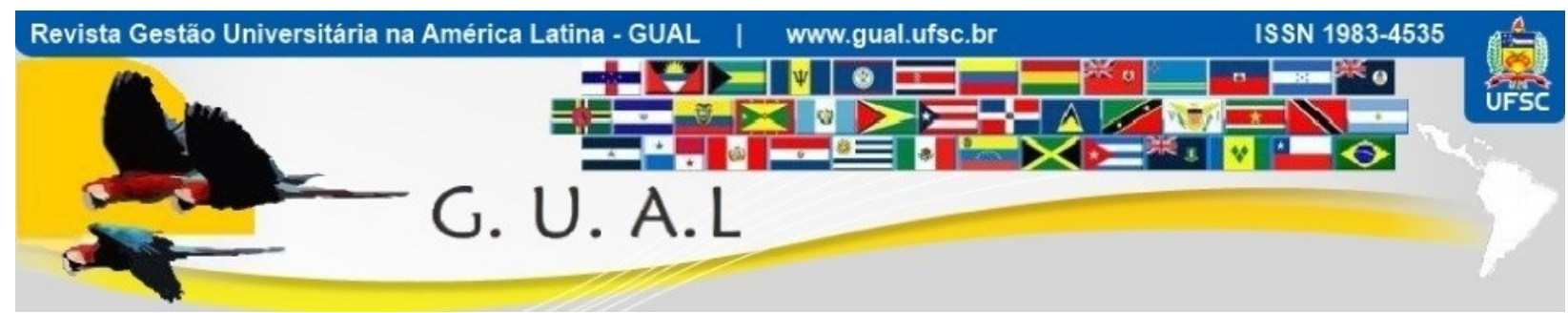

DOI: http://dx.doi.org/10.5007/1983-4535.2017v10n4p314

\title{
ESTUDIANTES: SUS PERCEPCIONES SOBRE LA CALIDAD EDUCATIVA Y SU IMPORTANCIA PARA EL COMPROMISO SOCIAL UNIVERSITARIO
}

\section{STUDENTS: THEIR PERCEPTIONS ABOUT EDUCATIONAL QUALITY AND ITS IMPORTANCE FOR UNIVERSITY SOCIAL COMMITMENT.}

Elia Marúm Espinosa, Doutora Universidad Nacional Autónoma de México - UNAM eliamarume@yahoo.com.mx

Federico Curiel Gutiérrez, Graduado Universidad Nacional Autónoma de México - UNAM fcuriel@cucea.udg.mx

Víctor M. Rosario Muñoz, Doutor Universidad Nacional Autónoma de México - UNAM vrosario14al18@hotmail.com

Recebido em 25/novembro/2016

Aprovado em 16/outubro/2017

Sistema de Avaliação: Double Blind Review

Esta obra está sob uma Licença Creative Commons Atribuição-Uso. 


\title{
RESUMEN
}

Los resultados de la investigación sobre la percepción de la calidad que tienen los estudiantes en México y como perciben que es esta adhesión en la universidades donde se forman, se estudia como parte del proyecto de largo aliento impulsado por la red ECUALE (Estudios sobre la Calidad de la Universidad en América Latina y España), donde ser realizó la investigación para docentes y para directivos y gestores. El estudiantado mexicano de pregrado (licenciaturas), se adhiere mas a conceptos de calidad modernos, asociados a su transformación, que a conceptos tradicionales o empresariales asociados al cumplimiento de objetivos y estándares, pero ven menos clara la adhesión de las universidades donde estudian a los conceptos con los que ellos mas se identifican, lo que da evidencias que pueden integrarse en la gestión institucional y en las políticas públicas e institucionales para el mejoramiento de la calidad en las universidades.

Palabras-clave: Estudiantes. Calidad Educativa. Gestión de la calidad. Percepciones de la calidad. Estudiantes y compromiso social.

\begin{abstract}
The results of the research on the perception of quality that students have in Mexico and how they perceive that this adhesion is in the universities where they are formed, is studied as part of the long-term project promoted by the ECUALE network (Studies on Quality from the University in Latin America and Spain), where research was carried out for teachers and for managers. Mexican undergraduate students (bachelor's degrees), adhere more to modern quality concepts, associated with their transformation, than to traditional or business concepts associated with meeting objectives and standards, but see less clearly the adherence of the universities where they study, to the concepts with which they are most identified, which gives evidence that can be integrated into institutional management and into public and institutional policies for the improvement of quality in universities.
\end{abstract}

Keywords: Students. Educational Quality. Quality Management. Perceptions of Quality. Students and Social Commitment. 


\section{INTRODUCCIÓN}

Si bien el gobierno (governance) y la gestión (management) de las instituciones de educación superior (IES) son funciones independientes y propias de dos campos distintos del conocimiento, tienen estrechas relaciones e interacciones múltiples y en la actualidad los límites entre los conceptos de management y governance de acuerdo con Sánchez y López (2013), aparecen cada vez más desdibujados. La literatura consigna que si bien "la relación entre la gobernanza y la gestión es un proceso reelaborado a diario por quienes tienen responsabilidades en la actividad institucional, desde los que ostentan mayor responsabilidad hasta los equipos docentes" (Whitchurch y Gordon, 2011; 67), no podemos abordarlo sin considerar que quienes son estudiantes son también parte de este proceso, ya que el estudiantado no solo es copartícipe y corresponsable de su proyecto formativo en procesos centrados en el aprendizaje, y por lo tanto cogestor de su formación, sino que también en nuestros países, forma parte del gobierno de las instituciones en todos sus niveles y por ello se convierte en un actor central del proceso.

La reelaboración cotidiana del proceso gestión-gobierno en las instituciones de educación superior (IES) se diferencia de la que se realiza en organizaciones empresariales de donde provienen muchos de los referentes teóricos que prevalecen hasta la fecha en las IES, producto de la irrupción en las universidades del llamado nuevo gerencialismo (New Public Management), enfoques y principios propios de las empresas privadas (lógica del mercado), y de los que señalan las propuestas y modelos para el gobierno, pues en la gobernanza y la gestión universitaria existe la necesidad de integrar de manera equilibrada los elementos del modelo educativo y académico, con las estrategias didáctico-pedagógicas y con las de índole organizativa y de gestión, donde, como ya señalamos, se ubica también al estudiantado.

En la investigación que realizamos sobre la percepción de la calidad y los factores que la determinan en mayor o en menor medida, de acuerdo con estudiantes de todo el país, reconocemos que el primer y principal compromiso social de la universidad es con quienes son estudiantes, y dentro de la gestión de la investigación deben considerarse con la prioridad que representan, a través de conocer, con líneas y proyectos de investigación, cómo perciben la calidad educativa y los procesos de gestión de esta calidad, que tiene una centralidad en las políticas públicas mexicanas.

La importancia de conocer la voz de las y los estudiantes sobre la calidad educativa para poder contar con evidencias e información que se integren a la retroalimentación de las 
políticas institucionales y públicas para su mejoramiento, han sido señaladas, entre otros por Leonid Grebennikov \& Mahsood Shah (2013), Kevin M. Elliott \& Margaret A. Healy (2001), Zepke, N. (2014). Los procesos centrados en el estudiante, el clima o ambiente de estudio en el campus y la capacidad y eficiencia instruccional de sus profesores tienen un fuerte impacto en la calidad, como lo afirman Kevin y Healy (2001). Estos autores sugieren también que las estrategias de ingreso deben hacer énfasis en diferentes experiencias de sus aspirantes, así como en estrategias para evitar su deserción, por lo que proponen que las universidades deban conocer las expectativas de sus estudiantes para atraerlos y retenerlos, así como saber que aspectos de su formación influyen más en su satisfacción. Ya en el año 2000, la UNICEF había reportado que luego de haber hecho una amplia revisión de la literatura sobre calidad educativa, se demostraba que los programas de estudio deben incluir definiciones amplias que incluyan aprendizajes, contenidos, procesos, ambientes y logros para sus estudiantes.

En la búsqueda de referentes que nos permitieran antecedentes para un mejor acercamiento a cómo perciben la calidad educativa quienes son estudiantes, nos encontramos con la situación que Kember, David, Doris y Leung, ya habían reportado en 2009, al afirmar que hay pocos instrumentos disponibles para evaluar la percepción de quienes son estudiantes sobre la docencia y ambientes de aprendizaje universitarios, y que estos instrumentos pueden proveer diagnósticos que retroalimenten a los programas de grado (licenciaturas o titulaciones) sobre la calidad del ambiente en que se desarrollan.

Estos autores aplican un cuestionario con escalas que buscan retroalimentación de las y los estudiantes sobre 9 facetas de la docencia y el ambiente de aprendizaje y sobre la percepción de la influencia que este ambiente tiene en el desarrollo de 8 capacidades o competencias genéricas definidas por el profesorado, para dar elementos para planes de acción que mejoren la calidad (Kember, David, Doris Y. P. Leung, 2009), pero no indagan sobre las adhesiones del estudiantado a conceptos de calidad, uno de los objetivos de nuestra investigación.

Otros autores analizan las actitudes hacia la calidad en instituciones de educación superior como elemento clave del sistema de gestión, sistema que "se centra en establecer los estándares para los programas, como objetivos y mecanismos para lograr resultados de aprendizaje y contribuir al nivel de resultados obtenidos, definidos como calidad de los estudiantes. Aquí, desde una perspectiva interna, el diseño curricular con sus recursos, la dedicación de sus docentes y de sus estudiantes, llevan a la calidad de las experiencias 
formativas de estudiantado, que debe ser apoyada por servicios y estándares establecidos para el programa" (Hrnčiar, Miroslav \& Peter Madzík, 2015; p.7).

La calidad educativa y en especial la calidad de la educación en el nivel superior ha sido estudiada como parte de la investigación sobre percepciones de la calidad de la universidad en América Latina y Europa por el grupo que forma la red ECUALE (Estudios sobre Calidad de las Universidades en América Latina y España), del cual formamos parte y donde se ubica esta investigación sobre el estudiantado mexicano y sus percepciones sobre la calidad educativa de su formación.

Hasta ahora no existía en México ningún estudio de estas características en cuanto a amplitud y profundidad. Podríamos enmarcar esta investigación en la necesidad muchas veces proclamada y pocas atendida de dar la voz al alumnado en cuestiones de tanta importancia. Investigaciones anteriores (Cardona, 2011,Olaskoaga, Marúm, Rosario y Lechosa 2013; Marúm, Rosario, Coords. 2015), también pioneras en su género, recogen la voz del profesorado y de directivos y gestores. Los resultados presentados aquí son parte del proyecto que complementa y en algún modo completa una visión de la calidad desde el punto de vista de los principales actores, de quienes de verdad saltan al ruedo de la educación: profesores y estudiantes, haciendo énfasis en este último.

\section{LA CALIDAD EM LA EDUCACIÓN SUPERIOR}

Existen desafíos y estrategias para definir la calidad en la educación superior, Schindler, L., Puls-Elvidge, S., Welzant, H., \& Crawford, L. (2015) encuentran 4 conceptualizaciones de calidad, calidad como intencionalidad, como excelencia o excepcionalidad, como transformación y como rendición de cuentas. A pesar del número de publicaciones sobre calidad educativa en los últimos 20 años, los significados de la calidad de la educación superior han permanecido relativamente estables y coinciden con las desarrolladas originalmente en los noventa (Harvey \& Green, 1993; Harvey \& Knight, 1996).

Actualmente, señalan Schindler, et al (2015), hay una tendencia a que sean los grupos de interés (stakeholders) quienes definan la calidad y ya no agencias externas basadas en estándares. Las y los estudiantes son uno de los grupos de interés más importantes para la educación superior, por lo que se ubica en este planteamiento la necesidad de conocer sus percepciones sobre la calidad y su opinión sobre los factores más importantes, y los que lo son menos, para lograr la mejora de la calidad de su formación. 
Retomaremos a continuación los resultados y algunos aportes de investigaciones que han precedido a la presente, para ubicar la conceptualización de calidad de la educación superior y la operacionalidad que de ella hemos hecho en trabajos previos para dar un marco conceptual de la calidad educativa.

Con toda probabilidad, señalan Olaskoaga, Marúm y Partida (2015), la tipología de nociones de calidad de mayor éxito en la literatura es la propuesta por Harvey y Green en 1993: calidad entendida como algo excepcional, como perfección o consistencia, como adecuación a una finalidad, como transformación. Esta tipología reconoce abiertamente la condición polisémica del término calidad cuando se aplica a la Educación Superior. En su trabajo seminal Olaskoaga, Marúm y Partida (2015) formularon cinco acepciones diferentes para la misma expresión, que a continuación se resumen de acuerdo con las aportaciones de Barrenetxea (2005).

Trabajos con una perspectiva más empírica (Barandiaran et al., 2012; Marúm et al., 2011a; Mijangos et al., 2011; Watty, 2006, 2005; Lomas, 2002) han puesto de manifiesto que si hay alguna noción de calidad que los académicos prefieren sobre las demás, ésa es la de la transformación del estudiante que destaca su propio papel en el proceso educativo, además del que le corresponde al estudiante. Olaskoaga et al. (2011 y ver también en Barandiaran et al., 2012; y Mijangos et al., 2011) han propuesto una reordenación de los conceptos de calidad de Harvey y Green que, a su entender, clarifica el sentido que adquieren dichos conceptos para quienes son académicos y que hemos utilizado para esta investigación sobre estudiantes.

\footnotetext{
1. La calidad como condición excepcional. Esta noción interpreta la calidad como algo exclusivo, que se establece en términos absolutos y se atribuye sin debate a una pocas instituciones, o bien la vincula a la excelencia en el sentido de superación de determinados estándares en los inputs o en los outputs de la educación superior: profesores, alumnos, equipamientos científicos, calificaciones de los alumnos, etcétera.

2. La calidad como perfección o consistencia. Desde este punto de vista la calidad se concibe como la reducción de la probabilidad de defectos en el producto, hasta su eventual eliminación.

3. La calidad como adecuación a una finalidad. Es la noción de calidad que predomina en la gestión empresarial de las últimas décadas. De acuerdo con este planteamiento, el servicio prestado por una IES se considera de calidad si cumple con las expectativas de algún cliente o stakeholder.

4. La calidad como entrega de valor por dinero (value for money). Aquí la calidad se describe como eficiencia en el uso de los recursos empleados. Es el planteamiento de los agentes financiadores de la Educación Superior, y que se ha difundido de la mano de la Nueva Administración Pública.

5. La calidad como transformación. Es la visión por la que se decantan los propios Harvey y Green. La transformación que se sugiere debe entenderse, como dicen Wittek y Kvernbekk (2011:674), en el sentido del término "bildung", que se emplea en la filosofía de la educación para referirse a un proceso de maduración personal de carácter integral. Harvey y Green lo expresan diciendo que "la educación no es un servicio para el cliente, sino un proceso continuo de transformación de los participantes" (Harvey y Green, 1993:24) en el que a sus estudiantes les corresponde el papel principal.
} 
Tabela 1 Conceptos de calidad de la educación superior

C1: La calidad consiste en desarrollar las capacidades del estudiante y en posibilitarlo para influir en su propia transformación

C2: La calidad consiste en formar estudiantes capaces de asumir un compromiso social

C3: La calidad consiste en el cumplimiento de los objetivos educativos que ha establecido la universidad

C4: La calidad consiste en satisfacer las expectativas de todos los involucrados en la educación superior

C5: La calidad consiste en satisfacer los requerimientos de las organizaciones donde se colocan los egresados

C6: La calidad consiste en el cumplimiento y la mejora de estándares establecidos

C7: La calidad consiste en conseguir trabajar con eficiencia: que la universidad cumpla con sus funciones pero al menor costo posible

Fuente: Adaptado de Mijangos, et al, 2011.

De definiciones de calidad, la primera y seguna (C1 y C2) asociadas a la transformación del estudiante y a la adecuación a una finalidad las hemos denominado concepciones modernas, y el resto han sido consideradas como tradicionales.

Para esta investigación hemos utilizado el enfoque sintetizador de los datos obtenidos en los estudios realizados con docentes (DOCALE), que resulta de la agrupación de los siete conceptos de calidad propuestos en tres componentes o dimensiones que se caracterizan por la forma de entender la calidad:

- Poder transformador de la educación universitaria

- Expectativas de los involucrados

- Consecución de los objetivos de la organización

De forma resumida, asociamos la primera de las dimensiones con una visión más pedagógica que identifica la calidad con el poder transformador de la educación; la segunda categoría está relacionada con la satisfacción de las expectativas de las partes involucradas: estudiantes, empleadores, etc.; y la tercera categoría hace referencia a la eficacia y la eficiencia en el cumplimiento de los objetivos y la misión de las universidades.

La diversidad de sentidos y matices que pueden darse a la calidad de la educación superior queda suficientemente demostrada apelando a la conceptualización de Harvey y Green (1993) y los desarrollos de Olaskoaga et al. (2011) y Barandiaran et al. (2012); pero para afirmar que los distintos actores se adhieren a diferentes conceptos de calidas según la posición que ocupan en la institución, esto es, que existe un contenido político de la noción de calidad, es decir, es preciso demostrar que "las preferencias por las nociones no se distribuyen 
homogéneamente entre los agentes que actúan en el sector, es decir, que existe un conflicto, real o imaginado, visible o latente, entre percepciones distintas de lo que debería ser la universidad" (Olaskoaga, et al, 2015). Este conflicto aparente entre las concepciones diferentes que tienen quienes pertenecen al profesorado y quienes son directivos o gestores, persiste también entre las percepciones estudiantiles y las de quienes son directivos y gestores, aunque no existe diferencia significativa con la de quienes son docentes.

\section{OPNIONES DEL ESTUDIANTADO SOBRE SUS CONCEPTOS DE CALIDAD Y LOS QUE PERCIBEN EM SUS UNIVERSIDADES. ANÁLISIS DE LOS RESULTADOS DE LA ENCUESTA A ESTUDIANTES DE PREGRADO}

Las opiniones recogidas en la muestra de estudiantes de licenciatura, nos permiten conocer tanto la percepción que éstos tiene del concepto de calidad de la educación superior, como su propia opinión sobre lo que conciben como el concepto de calidad educativa prevaleciente en la universidad en la que estudian.

En el cuestionario que se pasó a estudiantes de pregrado se les solicitaba que hicieran dos valoraciones sobre los conceptos de calidad, a través de los enunciados con los que hemos operacionalizado éstos. La primera de las valoraciones era sobre su grado de acuerdo con cada uno de esos enunciados. La segunda, valoraba el grado en que las y los estudiantes percibían que sus universidades se adherían a cada una de las afirmaciones.

En ambos casos, para la valoración, se pedía a quien se encuestaba, situara su grado de acuerdo en una escala de 5 categorías, desde "muy bajo" a "muy alto". En nuestro caso, y al igual que en investigaciones anteriores sobre profesorado y gestores y directivos, utilizaremos un indicador que a partir de ahora denotaremos como FRA, que es la suma de las frecuencias relativas de las respuestas "muy alto" y "alto". Este indicador, aparte de los requisitos mencionados, nos permite la comparación de los resultados con los de las investigaciones precedentes mencionadas .

En la ilustración 1 pueden verse los valores del indicador FRA. 


\section{Ilustración 1.Adhesiones a conceptos de calidad educativa estudiantes de licenciatura}

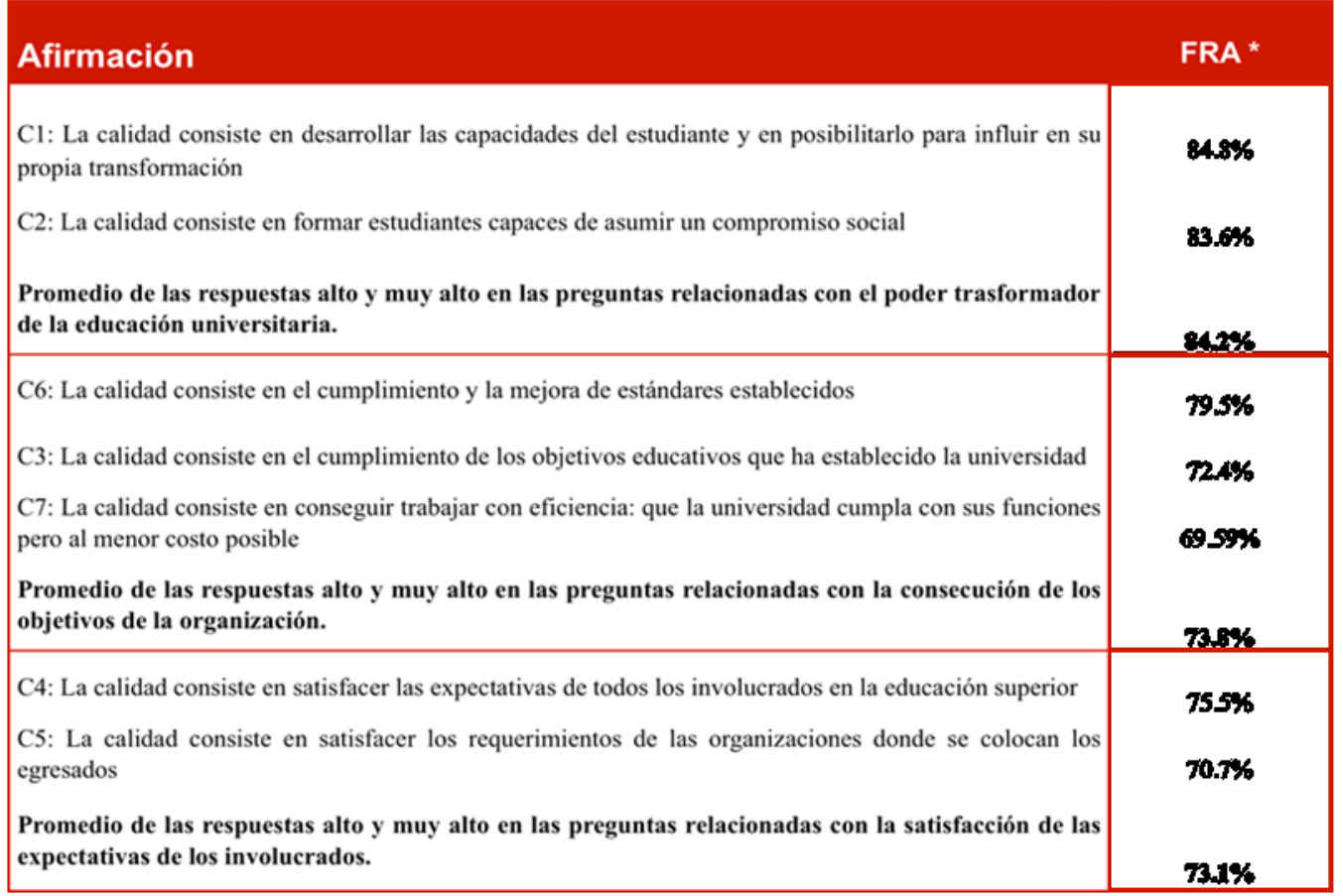

Fuente: Elaboración propia con base en los resultados de la encuesta.

Una primera conclusión que sacamos de las opiniones mostradas en la ilustración 1, es que quienes son estudiantes se identifican con concepciones modernas de calidad asociadas al poder trasformación de la educación superior, y en particular, la opción menos valorada es precisamente la más tradicional que puede entenderse como la más cercana a una concepción "empresarial" de la calidad: obtener más a menor costo y responder a las necesidades de los empleadores. En esto hay una gran coincidencia con las percepciones del profesorado.

Como ya se ha mencionado, quienes fueron encuestados no sólo respondían acerca de su opinión sobre la calidad educativa, sino que se les solicitaba que expresaran su percepción sobre la concepción de esa calidad que consideraban era la asumida por las universidades en las que estudian.

Los resultados de la comparación entre las concepciones de los individuos y las atribuidas a las instituciones, nos permitirán detectar la existencia o no de conflicto entre los estudiantes y las políticas de calidad percibidas por éstos en sus universidades.

La Ilustración 2 nos muestra los resultados del indicador FRA para las preguntas relativas a la concepción atribuida a las universidades. Al igual que en la Ilustración 1, se indica también el valor correspondiente a cada una de las tres dimensiones usadas para el 
análisis, calculado como media de los valores de los enunciados asociados a la dimensión correspondiente.

Ilustración 2 Conceptos de calidad de estudiantes vs. conceptos atribuidos a su universidad.

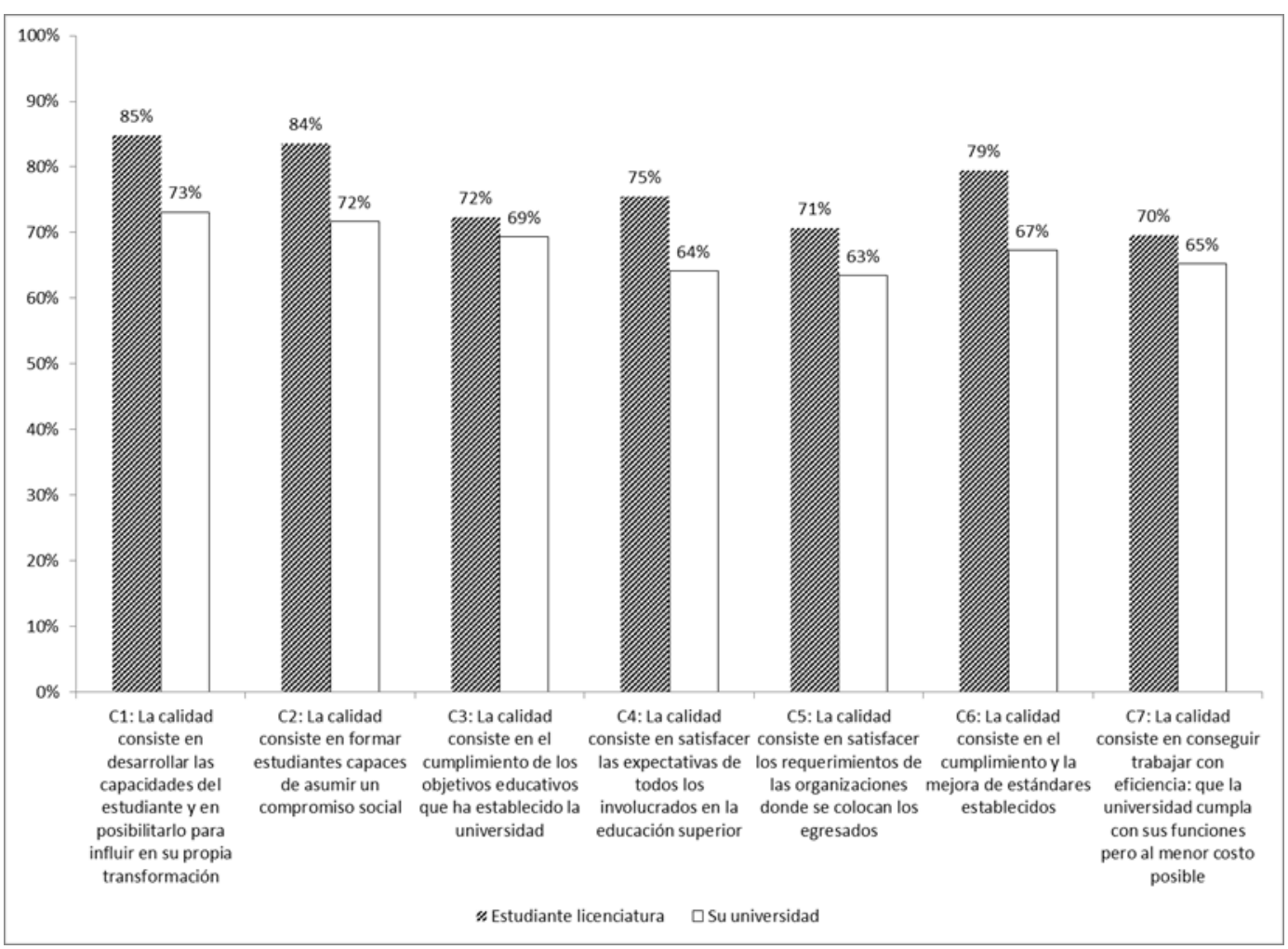

Fuente: Elaboración propia con base en los resultados de la encuesta.

Los conceptos de calidad se han ordenado de acuerdo con las tres dimensiones y según el grado de adhesión a cada grupo. Dentro de cada grupo los conceptos también se han ordenado en función del grado de adhesión, medido a través del FRA, de mayor a menor. Una comparación entre la concepción declarada por los estudiantes y la que le atribuyen a sus universidades puede verse en la ilustración 2. Esta ilustración nos proporciona otra visión más sintética de la diferencias entre la adhesión a los conceptos de calidad y la atribución hecha a sus universidades. Observemos que la valoración de la adhesión de los individuos está en todos los casos por encima de la atribución a la institución. Esto podría deberse a que el estudiantado tiene más clara su adhesión que la atribución; la distancia entre la concepción de calidad de los colectivos y la atribuida a las instituciones no es muy grande para el alumnado y podemos observar cómo quienes son estudiantes tienen una visión más equlibrada de todos los conceptos de calidad, sin embargo existen diferencias entre los conceptos de calidad a los que se adhiere el estudiantado y aquellos que consideran son los que prevalecen en las 
instituciones donde se forman, lo cual debe analizarse con mayor detalle y considerarse para la gestión de las universidades, ya que el estudiantado no coincide, con el mismo énfasis en las concepciones que prevalecen en sus instituciones .

Entre la adhesión de docentes a las concepciones de calidad y la que tienen quienes son estudiantes hay una clara coincidencia ya que los dos colectivos dan un mayor valor a los conceptos asociados a la calidad como transformación; mientras que en los conceptos asociados con la eficiencia, el cumplimiento de estándares y la satisfacción de los futuros empleadores es donde los estudiantes se distancian claramente, dando valores superiores a los otorgados por el profesorado. Esto nos puede llevar a pensar que las y los estudiantes mexicanos se ven más como clientes y financiadores de la institución y ven a ésta como suministradora de competencias para el mercado laboral, de lo que lo perciben quienes son profesores.

\section{CONCLUSIÓN}

El estudiantado muestra una adhesión a los conceptos de calidad, y percibe que hay menos énfasis en sus universidades hacia los conceptos de calidad con los que se identifican. A su vez, quien es estudiante de pregrado en México coincide mas con las concepciones de calidad del profesorado que con las de quienes son gestores o directivos. Puede decirse que para las y los alumnos, la universidad es un medio para lograr sus objetivos: transformación, compromiso social u obtención de empleo, y a la vez le piden que cumpla con aquello que la universidad les ofrece y sea eficiente en el uso de los recursos. Sin embargo, el profesorado parece menos preocupado por satisfacer a agentes externos, cumplir objetivos que ven ajenos a ellos y sus alumnos, o buscar la eficiencia económica.

Este trabajo aporta evidencias para la gestión institucional de la calidad y para la mejor comprensión de uno de los actores centrales del proceso educativo: sus estudiantes

\section{REFERÊNCIAS}

BARANDIARAN, M. EI concepto de calidad en la educación superior, una estrategia de apoyo desde las universidades a la generación de desarrollo humano. Tesis doctoral. Universidad del País Vasco/Euskal Herriko Unibertsitatea UPV/EHU. http://biblioteca2012.hegoa.efaber.net/registros/19517, 2013.

BARANDIARAN, M.; CARDONA, A. BARRENETXEA, M.; MIJANGOS, J.J.; y OLASKOAGA, J. . La opinión del profesorado sobre la mejora de la educación superior en España, ¿depende de su rama de enseñanza? Anuario de Investigación y Desarrollo 
2012, Centro de Investigación y Desarrollo de la Pontifica Universidad Católica del Ecuador, Esmeraldas Ecuador, PUCESE. http://www.researchgate.net/publication/265449517, 2013.

BARRENETXEA, M. Modelos de calidad y evaluación de la educación superior en la Unión Europea. Teis doctoral. Univerwsidad del Pis Vasco/Euskal Herriko Unibertsitatea. Bilbao, España, 2005.

CARDONA, A. (coord.). Calidad en la educación superior ¿Qué modelo y en qué condiciones? La opinión del profesorado en Argentina, España y México. Guadalajara: Editorial Universitaria, 2011.

HARVEY, L., y GREEN, D.Defining Quality. Assessment \& Evaluation in Higher Education, 18:1, 9-34, 1993.

HARVEY, L.; y KNIGHT, P.T. Transforming higher education. Buckingham, England: Society for Research in Higher Education \& Open University Press, 1996.

HRNČIAR, M.; y MADZÍK, P. A 3D view of issues of quality in higher education, Total Quality Management \& Business Excellence. http://www.tandfonline.com/loi/ctqm20, 2015

KEMBER, D.; y LEUNG, D.Y.P. Development of a questionnaire for assessing students' perceptions of the teaching and learning environment and its use in quality assurance. Learning Environ Res 12, 15-29. DOI 10.1007/s10984-008-9050-7, 2009.

KEVIN M. ELLIOTT \& MARGARET A. Healy. Key Factors Influencing Student Satisfaction Related to Recruitment and Retention. Journal of Marketing for Higher Education, Volume 10, 2001 - Issue 4. http://mc.manuscriptcentral.com/wmhe, 2001.

Leonid GREBENNIKOV \& Mahsood SHAH. Student voice: using qualitative feedback from students to enhance their university experience. Teaching in Higher Education 18(6). August 2013.

https://www.researchgate.net/publication/263591592_Student voice using qualitative feedb ack from students to enhance their university experience, 2013.

LOMAS, L. Does the development of mass education necessarily mean the end of quality?, Quality in Higher Education, 8:1, 71-79, 2002.

Marúm ESPINOSA, E; Curiel GUTIÉRREZ, F.; Rosario MUÑOZ, V.M., et al Estudiantes frente al espejo. Percepciones de la calidad educativa en programas de licenciatura y posgrado en México. México, Universidad de Guadalajara/CONACYT, 2015.

MARÚM, Rosario (Coord.). La calidad del pregrado y posgrado. Una mirada iberoamericana. México. Universidad de Guadalajara/Conacyt, 2015.

MIJANGOS, J.J. (coord.). Enseñanza Universitaria de Calidad: profesorado, Alumnado e Institución. Bilbao: Editorial de la Universidad del País Vasco/Euskal Herriko Unibertsitatea UPV/EHU, 2011. 
OLASKOAGA, J. (coord.). Hacia una educación superior de calidad. Un análisis desde la perspectiva del profesorado en Argentina, Chile, España y México. La Plata: Editorial de la Universidad Nacional de la Plata, 2009.

OLASKOAGA, J; MARÚM, E; ROSARIO; V y LECHOSA; D. Universidades en movimiento. EI debata acerca de la gestión de la calidad y las actitudes del profesorado ante las transformaciones univesitarias. México, ANUIES, 2013.

OLASKOAGA, J.; GONZÁLEZ, X.; y BARRENETXEA, M.. Political nature and socioprofessional determinants of the concept of quality, Higher Education. 69, 673-691, 2015.

OLASKOAGA, J.; MARÚM, E.; y PARTIDA, I. La diversidad semántica y el carácter político de las nociones de calidad en la Educación Superior de México, Revista de la Educación Superior, XLIV:1, 85-102, 2015

Sánchez MORENO, Marita y Julián López YAÑEZ .Buenas Prácticas de Gobierno y Gestión en la Universidad. Teoría Educativa 25, 1-2013. pp. 125-148. Recuperado el 22 de febrero de 2015 de:

https://www.google.es/\#q=banco+mundial+buenas+practicas+gestión+universidades

SCHINDLER, L.; PULS-ELVIDGE, S.; WELZANt, H.; y CRAWFORD, L. Definitions of quality in higher education: A synthesis of the literature. Higher Learning Research Communications, 5:3, 3-13, 2013

UNICEF . Defining Quality in Education .A paper presented by UNICEF at the meeting of UNICEF The International Working Group on Education Florence, Italy June. Working Paper Series Education Section Programme Division United Nations Children's Fund New York, NY, USA, 2000.

WATTY, K. Quality in accounting education: what say the academics?, Quality Assurance in Education, 13:2, 120-131, 2005.

WHITCHURCH, C. y GORDON, G Some Implications of a Diversifying Workforce for Governance and Management. Tertiary Education and Management, 17 (1), 65-77, 2011.

Wittek, LINE, and Tone KVERNBEKK. "On the Problems of Asking for a Definition of Quality in Education.” Scandinavian Journal of Educational Research, 55 (6): 671-84, 2011.

ZEPKE, N. Student engagement research in higher education: questioning an academic orthodoxy, Teaching in Higher Education, 19:6, 697-708.

http://www.tandfonline.com/loi/cthe20, 2014. 